\section{One hundred years of} diet-heart research: Good science, junk science and honest mistakes

\author{
Paul B. Addis ${ }^{1}$ and Martin Grootveld ${ }^{2 \star}$
}

${ }^{1}$ Professor Emeritus, Department of Food Science and Nutrition, University of Minnesota, 1334 Eckles Ave., St. Paul, MN 55108, USA

${ }^{2}$ Full Professor, Leicester School of Pharmacy, De Montfort University, The Gateway, Leicester LE1 9BH, United Kingdom

\begin{abstract}
Submitted: 29 August 2021
Approved: 23 September 2021

Published: 26 September 2021
\end{abstract}

Address for correspondence:

Prof. M. Grootveld, Leicester School of Pharmacy, De Montfort University, The Gateway, Leicester LE1 9BH, United Kingdom.

How to cite this article: Addis PB, Grootveld M. One hundred years of diet-heart research: The good science, junk science and honest mistakes. G Med Sci. 2021; 2(5): 001-013. https://www.doi.org/10.46766/thegms.nufosci. 21082908

Copyright: (c) 2021 Paul B. Addis, Martin Grootveld. This is an Open Access article distributed under the Creative Commons Attribution License, which permits unrestricted use, distribution, and reproduction in any medium, provided the original work is properly cited.

\section{Overview}

It is difficult to imagine an area of scientific research, over the past century, that has been more controversial than the diet-heart health question. Even more unsettling is the fact that the hypotheses presented in this scholarly review often directly conflict with or reverse prevailing opinions. For example, many brave researchers are fully aware that dietary polyunsaturated fatty acids (PUFAs) peroxidatively degrade to highly toxic lipid oxidation products (LOPs) such as chemically-reactive aldehydes when exposed to high-temperature frying, cooking or processing practices. Currently, many now also accept that LOPs arising therefrom are actually quite dangerous if ingested after the subjection of PUFA-rich frying oils to excessive heating in this manner, or following storage at ambient temperatures for significant periods of time, as is often the case. Moreover, even less-reactive monounsaturated fatty acids (MUFAs) are also susceptible to oxidative damage, especially if present in cooking oils reused for repeated frying episodes, or when subjected to prolonged storage periods, especially in bright light. As a corollary, we also believe that in view of their extreme stability, saturated fatty acids (SFAs) are far less dangerous than PUFAs, if used in this context. Similarly, we now recognize that the cholesterol-heart disease hypothesis was based on data obtained initially on severely flawed experiments. All indications are that 'pure', i.e., unoxidized, cholesterol is harmless; indeed, cholesterol is one of the most vital biomolecules present in the human body. This debate brings up the topics of "good science, junk science and honest mistakes." All three approaches will be discussed in the current paper. The ramifications of erroneous conclusions regarding PUFAs and cholesterol are enormous. Some aspects of these are further explained below, although in our opinion, they have indeed continued to exert detrimental effects upon human health.

This paper represents an opinionated review (i.e., a perspective). As such, it relies on both articles published in rigorously-reviewed, reputable research journals, along with valuable anecdotal information, precisely arising from the lengthy academic and commercially-relevant experiences of the authors.

\section{Cholesterol}

Likely, the very first studies implicating animal fats/ lipids in heart disease were performed by Anitschkow and colleagues $[1,2]$, who fed cholesterol to rabbits and identified the resulting detailed atherosclerotic lesions. Indeed, so successful was his group that hewas credited with developing the early standard protocol for atherosclerosis research involving cholesterol feeding. There are several essential issues in this research approach, especially if attempts were made, and they were, to relate it and its consequences in humans. Rabbits are herbivores, and therefore do not, like most humans, possess homeostatic mechanisms that control blood cholesterol. Rabbits, unlike humans, experience extremely high blood cholesterol 
levels if fed cholesterol. Furthermore, subsequent research has shown that the cholesterol used by Anitschkow was heavily contaminated with cholesterol oxidation products (COPs), which were later shown to be highly cytotoxic and atherogenic. It was also shown that pure cholesterol lacks the capacity to induce atherosclerotic plaque. A comprehensive treatise on the subject of cholesterol autoxidation and the differences in cytotoxicity of purified cholesterol and impure (oxidized) cholesterol was provided by Smith in 1981 [3]. The foregoing findings prompted a series of studies by our research group based in Minnesota, USA [4]. A comprehensive study of various foods identified three types that were problematic: tallow used for deep-frying in fast-food restaurants, freezedried "camper" foods, and powdered eggs processed by gas-fired spray dryers. Meat, fresh eggs, fish, cheese and other dairy products were basically free of COPs.

It is easy to be critical of Anitschkow's work [1, 2] and similar studies. However, at the time, oxygen-derived free radical species were largely unknown, as were COPs and other lipid oxidation products (LOPs). Therefore, this is an honest mistake, made possible by the lack of relevant knowledge at that time-point. It is also true that
Anitschow's work elucidated some of the critical steps in the atherosclerosis process. The misinterpretation of Anitschkow's research has caused countless cases of confusion and poor dietary recommendations. Moreover, credit where credit is due, but with the very exacting scientific standards developed, optimized and augmented many years later, with today's rigorous requirements would anyone sensibly rely on and attempt to further perpetuate research results originally reported more than a century ago [2]?

Fatty acids (FAs) also show a pattern parallel to cholesterol, namely native fatty acids not exhibiting cytotoxicity, but their corresponding LOPs such as conjugated hydroperoxydienes (CHPDs) and their aldehydic fragmentation products showing potent levels of such toxic actions. Beef is one important food that has been targeted for criticism, but in view of the high contents of peroxidation-resistant SFA and MUFA classes present, the FAs therein are amongst the most stable [5]. A schematic representation of the mechanisms of pathways involved in and secondary aldehydic LOPs arising from the lipid peroxidation process is shown in Figure 1.

\section{Figure 1}

(a)

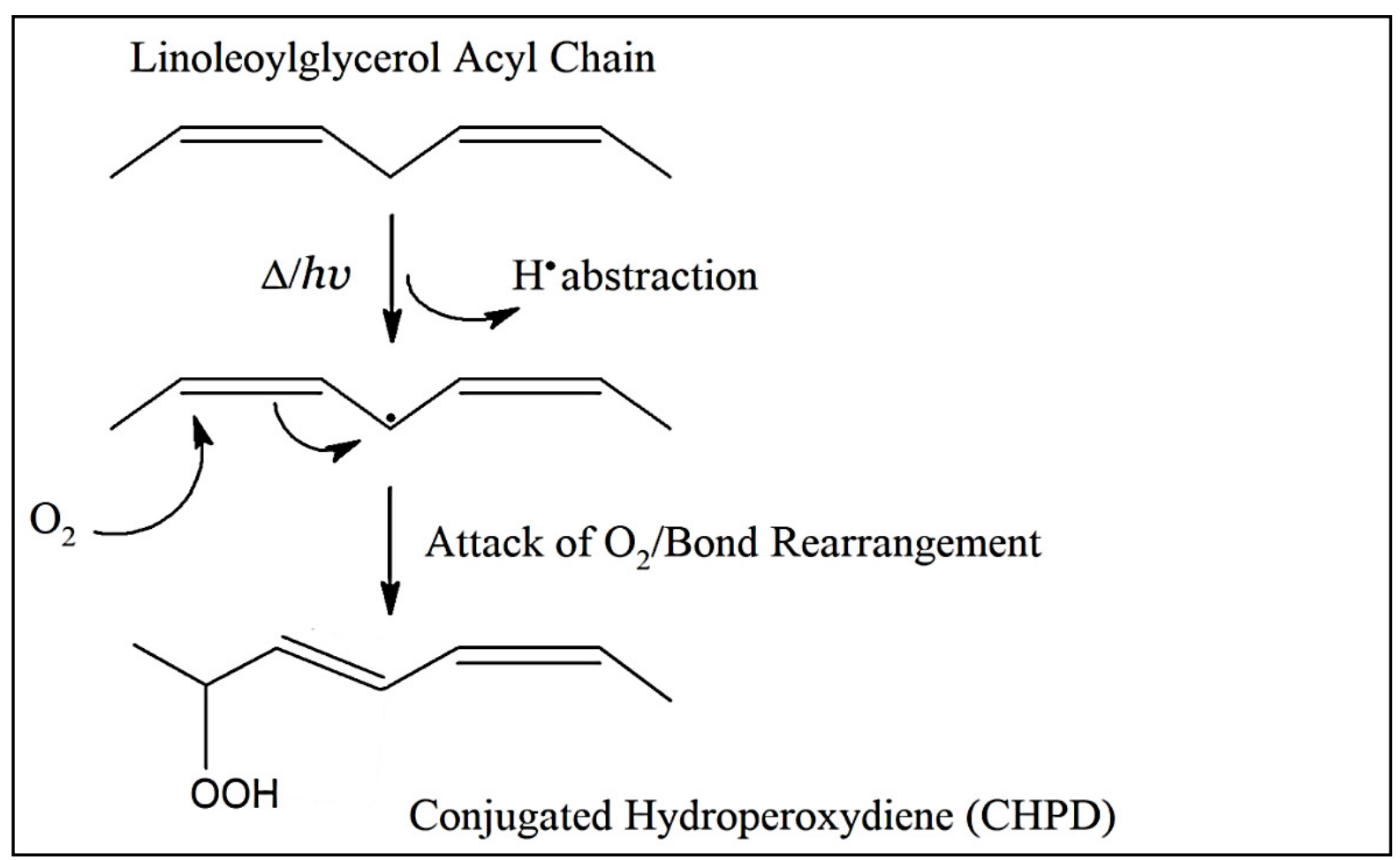


(b)

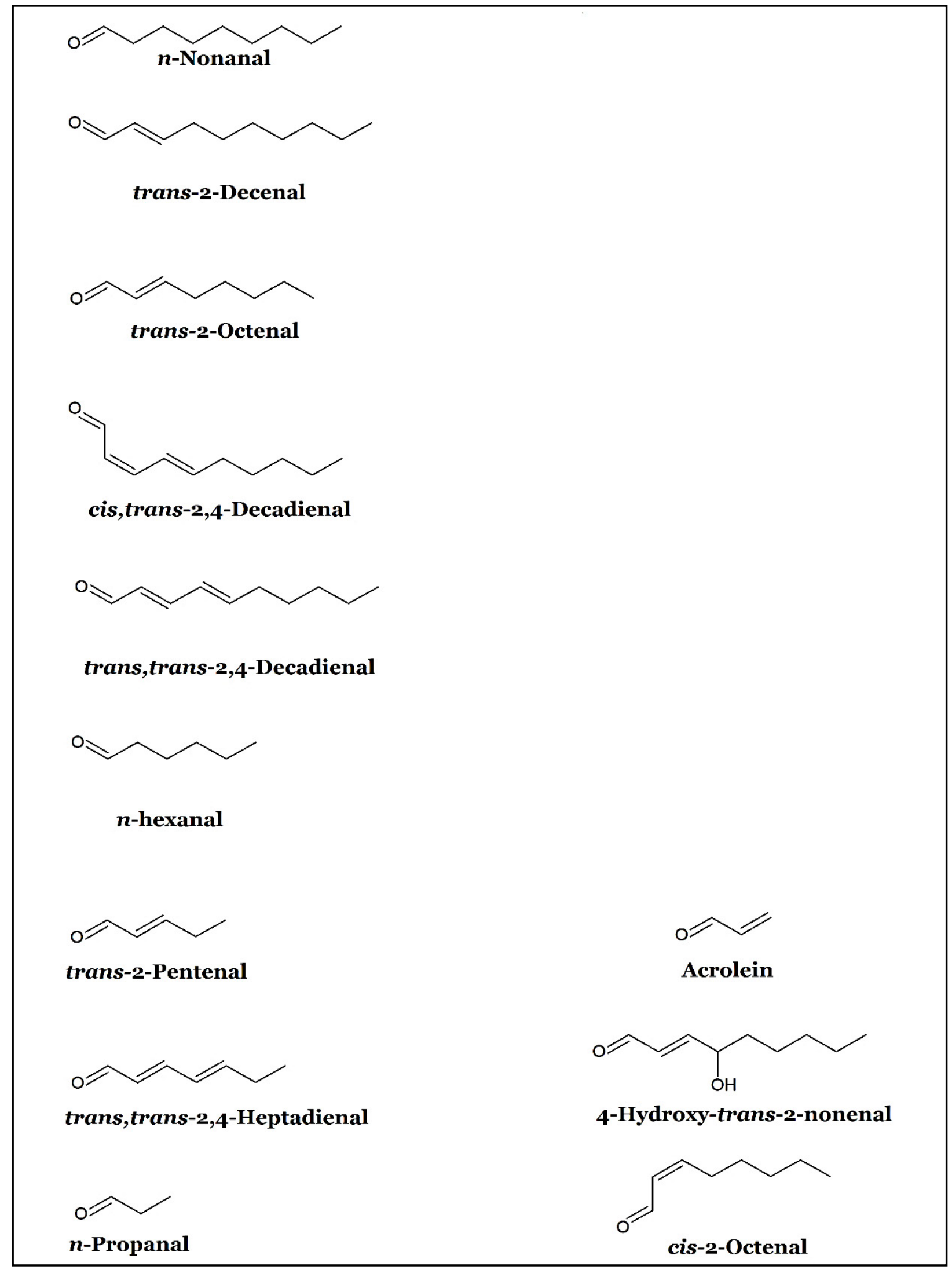

Figure 1. (a) Simplified reaction scheme for the peroxidation of a linoleic acid moiety present as a culinary oil triacylglycerol (TAG) acyl chain (H• represents a hydrogen atom). The conjugated hydroperoxydiene (CHPD) molecule shown has the cis,trans- configuration. (b) Molecular structures of aldehydes derived from the degradation of lipid hydroperoxides, specifically hydroperoxymonoenes (HPMs) from MUFAs, and CHPDs from PUFAs. $n$-Nonanal and 
trans-2-decenal arise from the fragmentation of oleoylglycerol-derived HPMs; $n$-hexanal, trans-2-octenal and trans, trans-deca-2,4-dienal from the fragmentation of linoleoylglycerol-derived CHPDs; and propanal, acrolein, trans2-pentenal and trans,trans-hepta-2,4-dienal from linolenoylglycerol-derived CHPD fragmentation. Acrolein can also be generated from oxidation of the glycerol backbone of TAGs, and cis,trans-deca-2,4-dienal may arise from the thermally-induced isomerism of its trans,trans-isomer, as may cis-2-alkenals from their corresponding trans-isomers (reproduced from Ref. [5] with permission).

\section{Fat and Cholesterol in Beef}

Beef has been the subject of much misinformation and taboo. It is commonly believed that beef fat is $100 \%(\mathrm{w} / \mathrm{w})$ saturated, and that beef is loaded with cholesterol. In actuality, beef fat is approximately $50 \%(\mathrm{w} / \mathrm{w})$ SFAs and $50 \%(\mathrm{w} / \mathrm{w})$ MUFAs, mostly oleic acid for the latter, the most famous FA of olive oil [6]. Furthermore, one of the principal SFAs in beef, stearic acid, is converted to oleic acid upon metabolic desaturation in the body. Cooking meat further lowers overall fat content. Therefore, it is challenging to visualize beef fat being responsible for elevated blood cholesterol. As discussed above, the small quantity of cholesterol in beef muscle, less than one-half of the amount in an equivalent weight of egg yolk, will not raise serum cholesterol. This topic will be elucidated in greater detail later in this review.

\section{Fish Oil Omega-3 FAs}

However, it was not just the egg and beef industries that were pummeled by erroneous data, conclusions and subsequent recommendations. Some promising technologies were buried too. After the initial excitement that fish oils created, some technology emerged that was able to remove cholesterol from such commodities, a very rich source of it for selected oil types. But the real benefit of this ultra-high vacuum distillation process was the deodorization of the oil, a process that removed virtually every trace of LOPs [7]. This fish oil exhibited amazing characteristics: no fishy flavor, little or no selfpropagating LOPs, no cholesterol, and an incredibly long shelf-life, especially when stored in the freezer. This was a great product, one poised to do a great deal of good for coronary patients everywhere. What could possibly go wrong? The answer is misinformation and a media feeding frenzy that followed.

The two main coronary-related properties of fish oil are lowered blood triacylglycerols (triglycerides) and, most importantly, reduced thrombogenic activity of blood platelets [8]. Blood cholesterol data was never as clear-cut. Nevertheless, a study published on the lack of cholesterol-lowering effects of fish oil was trumpeted by the media, the same people who were shouting the benefits of fish oils a year earlier. It essentially killed the fish oil market for years, but recently this has made a comeback. Unfortunately, many of the products sold to consumers are already peroxidised. They contain significant levels of LOPs, and consequently also somewhat reduced levels of the principal FAs potentially responsible for their positive health benefits [5]. CHPDs are also present in such oils. Moreover, most of these products have a fishy odour. Again, an over-emphasis of the importance of serum cholesterol did much more harm than good.

\section{Beef Tallow as a Frying Medium}

During this period, increased interest was developing world-wide on the potential significance of COPs. At the time, tallow, refined beef fat, was used for frying by a large number of fast-food restaurants. Our group published two papers on the oxidation of tallow cholesterol during deepfat frying practices $[9,10]$. These studies, together with the recognition of the toxicological properties of COPs, prompted the fast-food industry to switch to PUFA-rich oils (molecular structures of the most predominant COPs are shown in Figure 2). However, the opportunity existed to use the technology employed for deodorizing fish oil to remove cholesterol from the tallow. This technology worked magnificently, and a new frying oil was born. In removing cholesterol, traces of peroxides and other LOPs were also removed, so at the beginning of the frying cycle, LOP levels were negligible, an indication that little or no prior catalysis of lipid oxidation was likely. The result was a superb frying oil, which produced French fries of exceptional organoleptic quality. This oil also had a long oil fry life, contained virtually no LOPs, and also exhibited reduced absorption levels into fried foods. Potentially, this could have been the most refined frying oil ever produced, and was marketed to the fast-food industry. But alas, the stigma of saturated fat could not be overcome, and hence the finest frying oil died. How can the finest cooking oil fall prey to such misinformation? What's worse, the current practice of frying in restaurants is clearly more dangerous, and has been reviewed in detail [5]. How did this come to pass? The so-called "Seven Countries Study" [11-13] exerted a major impact. 


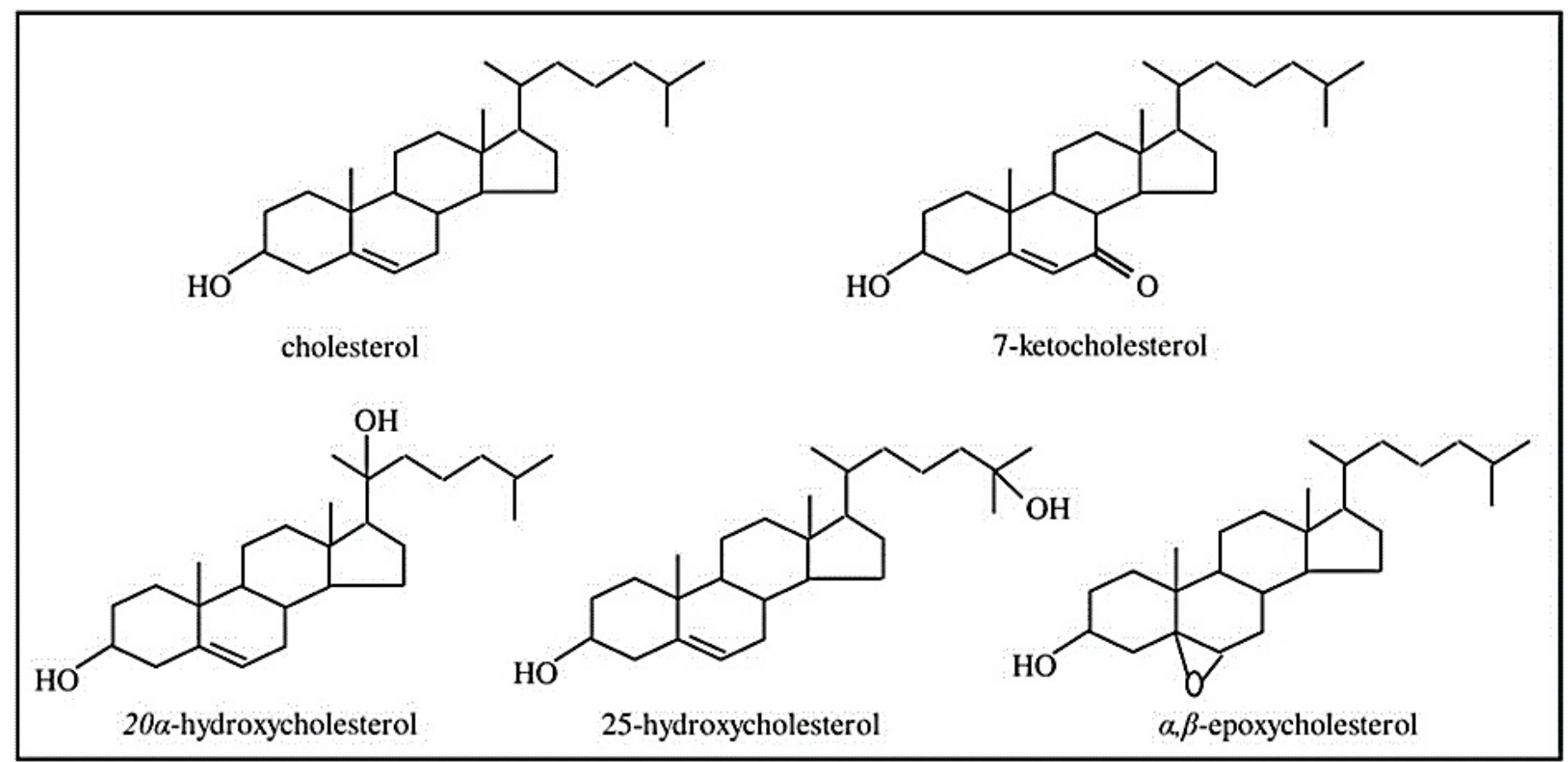

Figure 2. Molecular structures of cholesterol and its major oxidation products detectable in muscle foods. Reproduced from Ref. [14] with permission.

The author of the Seven Countries Study, Dr. Ancel Keys, was an outspoken researcher who had a difficulty comprehending viewpoints other than his own [15]. Keys was one of many medical researchers who employed epidemiology to establish a causal relationship between animal lipids and coronary heart disease. One serious issue featured in these studies is the cardinal rule of epidemiology: a statistical association does not establish causality. If this were not true, one could easily say, for example, that aspirin causes headaches. Most of these researchers also admit that statistical association cannot establish causality. They then make dietary recommendations based on their findings, often from poorly designed experiments [15]. No laboratory data were noted to support these epidemiology findings, and the statistical analyses applied was limited and problematical, for example difficulties encountered with the cross-classification of predictor risk factors, and their multicollinearity: Age, body weight, systolic blood pressure, dietary intakes of saturated fat and cholesterol, blood serum chlolesterol levels and cigarette smoking frequency/history parameters are predominantly significantly or even quite strongly inter-correlated with each other anyway. Therefore, it is not surprising that the media enthusiastically endorsed anti-animal fat statements. Teicholz [15] clearly outlined a number of flaws in several studies, including Keys' research. Although there remains a lot of blog- and scientific literature-based controversy over this issue, one common viewpoint on the Seven Countries Study is the careful, non-random and unstratified selection of 7 out of
22 countries originally surveyed, and that Keys allegedly ignored or removed those which failed to match his key hypothesis featuring the stigmatization of saturated fat and cholesterol intakes. Furthermore, the authors are also almost reluctant to even mention the 'Seven Countries' involved in the study in view of the availability of modernday web-based misinformation and confusion, but from the original report these were the USA, Finland, the Netherlands, Italy, Croatia (formerly Yugoslavia), Serbia (also formerly Yugoslavia), Greece and Japan, with a grand total of $>12,000$ study participants at the entry point. Consequently, there were two really unfortunate outcomes of this study that can only be known as "junk science."

Firstly, it was difficult to establish the fact that we should be concerned with a combination of LOPs and COPs. The prevailing publicity simply obscured laboratory results that were truly relevant. Secondly, as explained so expertly in Ref. [15], the dietary recommendations by governmental agencies did more harm than good. We now have rampant obesity and type 2 diabetes in the U. S., and also in other developed nations. An extremely high carbohydrate diet is certainly not a healthy one! [15].

Conversely, in 2015 de Souza et al. [16] reported on a systematic review and meta-analysis of observational studies, and found that saturated fat intake was unassociated with all-cause mortality, cardiovascular disease (CVD), coronary heart diseases (CHD), ischemic stroke, or type 2 diabetes, although the evidence available 
was somewhat heterogeneous, and hampered by a range of methodological limitations. However, trans-FA intake was found to be linked to all-cause mortality, total CHD, and CHD mortality, in view of the higher extents of industrial trans-FA consumption than that of their ruminant forms. Despite this, no considerations of the dietary intake of COPs, nor UFA-derived LOPs, were made in this study, which is often the case, even by today's standards.

\section{PUFAs in Frying Oils - Some Shocking Findings}

The foregoing discussion makes clear the importance of governmental regulatory agencies taking seriously, for the first time, the vast number of studies showing the contents of many types and homologues of toxic LOPs in frying oils and fried foods (Figure 3). Furthermore, recognizing the toxicity of LOPs, it must be emphasized that studies quantifying these toxins in foods, especially in frying oils, are numerous, detailed, and span several decades. One of the pioneers in this area was Prof. Hermann Esterbauer in Austria [17, 18]. His laboratory focused on aldehydes, including $\alpha, \beta$-unsaturated aldehydes. The $\alpha, \beta$-unsaturated aldehydes are highly reactive electrophilic alkylating agents and, as such, avidly seek out and react with nucleophilic sites in proteins, enzymes, deoxyribonucleic acid (DNA), and other biologicallycritical biomolecules. Esterbauer and many other workers have published scores of articles establishing and confirming the cytotoxicities and genotoxicities of LOPS.

Figure 3

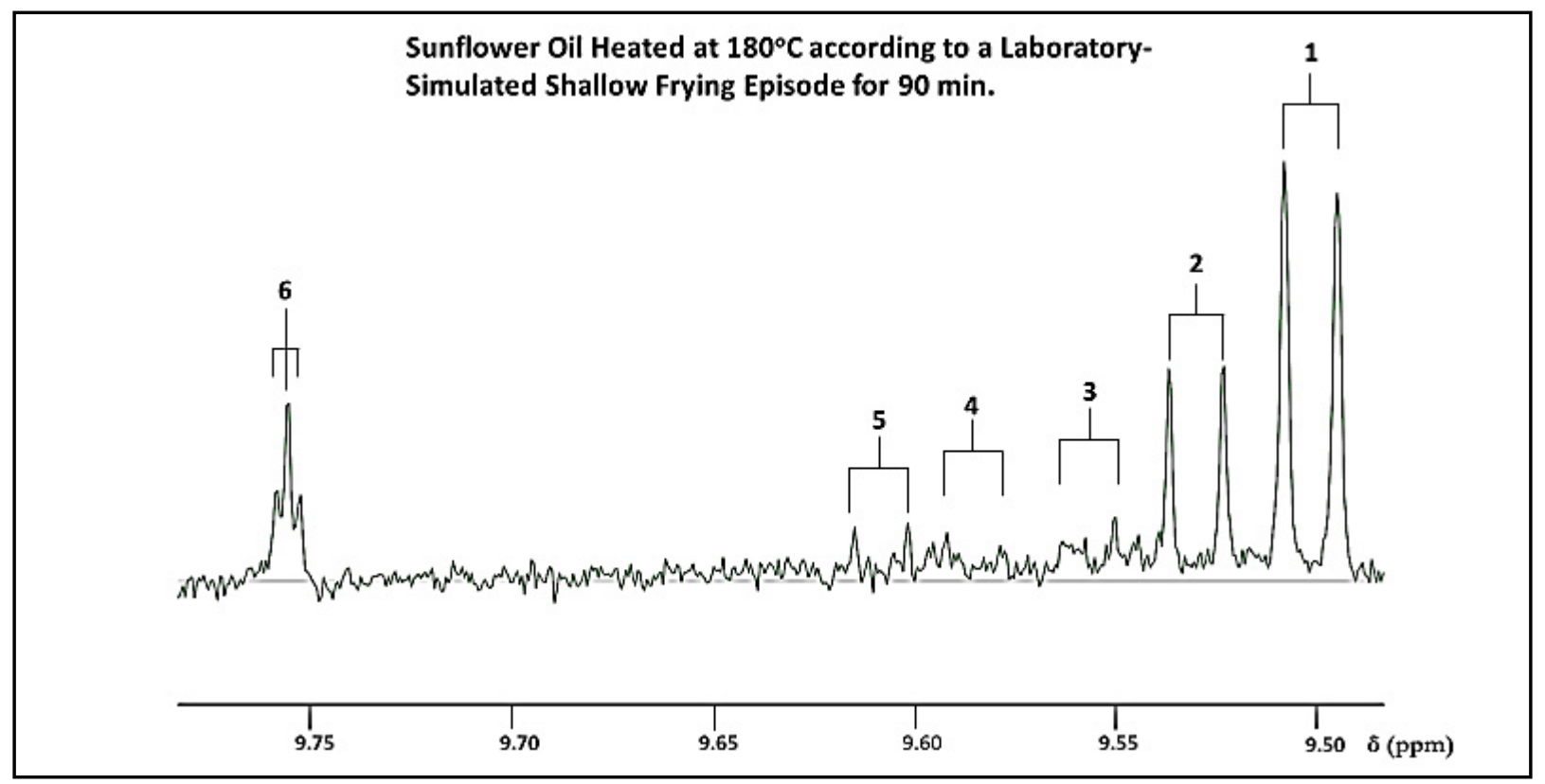

Figure 3. Aldehyde-CHO function region $\left(\delta=9.47-9.78 \mathrm{ppm}\right.$ ) of the $600 \mathrm{MHz}{ }^{1} \mathrm{H}$ NMR spectrum of a commerciallyavailable refined sunflower oil sample exposed to a laboratory-simulated shallow frying episode at $180^{\circ} \mathrm{C}$ for a period of $90 \mathrm{~min}$. Sample preparation and acquisition of ${ }^{1} \mathrm{H}$ NMR spectral profiles were performed according to a modification of the method reported in [19]. Assignments: 1, trans-2-alkenals; 2, trans,trans-alka-2,4-dienals; 3, 4,5-epoxy-trans-2alkenals; 4, 4-hydroxy-/4-hydroperoxy-trans-2-alkenals; 5, cis,trans-alka-2,4-dienals; 6, n-alkanals.

Perhaps the most dramatic toxicity findings of LOPs in heated oils was the teratogenic effects reported by Bonet and colleagues of Madrid, Spain [20], a development also reviewed by Grootveld et al. [21], in a journal serving the foodservice industry. The teratogenic effects noted translate to neural tube defects such as spina bifida.

The finding of teratogenic effects was shocking, but this did not slow the sales of French fries. However, there appears to be a trend for hot-air cooking of foods in recent years, a welcome alternative to frying.

The topics discussed in the preceding paragraphs have raised some serious concerns. Examples indicate that economic factors, not science, have influenced the results of research and dietary recommendations, neither of which have improved human health.

A review of atherogenic and anti-atherogenic factors in the human diet [22] would represent a balanced approach to making dietary recommendations. The atherogenic factors are LOPs (including COPs), trans-fatty acids, and some hypercholesterolemic saturated fatty acids. Antiatherogenic factors were identified as antioxidants, particularly Vitamin E [23]; fish oils and other PUFA sources, (but only if well protected against autoxidation); trace metal ion complexes, including those of iron (Fe), copper $(\mathrm{Cu})$, manganese $(\mathrm{Mn})$, zinc $(\mathrm{Zn})$ and selenium 
(Se); and fibre [19, 22]. Trace minerals are exceedingly essential since many, in particular those noted above, serve as cofactors for in vivo antioxidant systems such as the glutathione peroxidase enzyme. If used as the basis of dietary recommendations, the preceding information could better serve public health than the current timeconsuming method of developing recommendations.

\section{Dietary Recommendations - Can We Do Better?}

The extreme focus on dietary saturated fat and cholesterol as hazardous malefactor molecules, and replacement of the former with carbohydrates, has been detrimental to human health [24]. Cholesterol was first believed to be involved in heart disease because it always was present in atherosclerotic plaque. However, is this surprising given the fact that this agent is ubiquitous in living animal tissues? The human body biosynthesizes between 1,000 and 2,000 mg cholesterol per day. Nevertheless, this figure plummets if an individual increases their intake of cholesterol. Furthermore, the body converts more cholesterol into bile acids, eventually excreted, and reduces the former's absorption from dietary sources. It is very complicated to alter blood cholesterol levels via dietary means [25] - indeed, this is only possible up to a maximum of $c a .15 \%$ anyway. Furthermore, as far back as 1992, Apfelbaum [26] found that substitution of exactly one-half of the intake of regular butter by a cholesterolfree variety gave rise to a mere $0 \cdot 3 \%$ reduction in total blood cholesterol concentrations, which was 15-fold lower than the weekly physiological variation; this observation gave rise to much concern regarding the 'health benefits' putatively offered by this food product.

At the height of the cholesterol controversy, in 1968 the American Heart Association (AHA) recommended that human subjects consume no more than three eggs per week [27]. This policy was included in a range of dietary guidelines provided to the US public, along with further national recommendations, most especially those of the National Cholesterol Education program. In view of this complete misconception, for 50 years or so US citizens were strongly cautioned to limit their intakes of dietary cholesterol to $<300 \mathrm{mg}$ per day in order to facilitate the lowering of their blood plasma cholesterol levels and hence alleviate heart disease risks [27]. Was any harm done? Eggs represent one of the most essential packages of nutrients known to man, and also one of the least expensive. Egg white protein is nature's best. Additionally, the yolk is full of fat-soluble vitamins and also is a good source of minerals. Many elderly people could not afford more expensive sources of protein. Recent studies have demonstrated the beneficial health effects of egg consumption, including those focused on the monitoring of plasma high- and low-densitylipoprotein cholesterol and antioxidant concentrations in healthy young adults [28]. Eventually, in 2015, after many studies demonstrating the futility of attempting to modify serum cholesterol levels via dietary cholesterol manipulation, this $\mathrm{AHA}$ recommendation was revised to no recommendation at all for most human subjects [27].

Similarly, recommending a severe drop in the percentage dietary fat consumption has led to a significant increase in type 2 diabetes incidence. Correspondingly, one notable observation was that participants consuming a Mediterranean diet, which was supplemented with MUFArich extra-virgin olive oil, gave rise to a $52 \%$ decrease in diabetes incidence when compared with an appropriate control group [29].

Moreover, in 2014, Forouhi et al. [30] explored prospective associations between a range of objectively determined specific phospholipid SFAs and incident type 2 diabetes in a very large number of participants recruited to the EPIC-InterAct study, and for this purpose they conducted a longitudinal case-cohort investigation. Intriguingly, these researchers found that whereas even-numbered chain SFAs were positively associated with this condition, their odd-numbered chain forms were inversely associated with it. These data suggest that modifications in dietary patterns may indeed exert multifaceted health benefits over and above CVD and CHD. Stearic acid (C18:0) acts as a precursor to the "health-friendly" C17:0 SFA.

Finally, although difficult to quantify, how much harm has been done to people consuming foods fried in PUFArich culinary oils? Clearly, it is critically important that this issue is addressed as soon as possible. Currently, the nature and extent of such adverse health effects induced by the ingestion of dietary LOPs are not easily estimated in view of limitations of global datasets available, in particular those regarding population intakes of fried foods and other dietary LOP and COP sources. However, once such comprehensive datasets do become available, they will serve to facilitate estimates of the intakes of these toxins, which may then permit evaluations of their roles in the induction, development and progression of chronic non-communicable human diseases (CNCDs). Hence many further investigations are required, including powerful epidemiological cohort studies conducted across differing human populations, which specifically take into account the nature and extent of LOP-containing food intakes from a public health perspective, and which also critically consider in some detail the precise molecular structures of unsaturated fatty acid (UFA) and SFA species present within cooking oils, unused, used or reused, for the purpose of frying foods. Nevertheless, one major limitation of prospective public health-related epidemiological studies is the extensive periods of time required for their completion, which may often stretch to many years or even decades. Notably, to date, recommendations that restricted cholesterol and saturated fat consumption in view of their "proven" harmful effects on human health 
have certainly not offered any demonstrable benefits [24].

\section{Recognition of the Importance of Dietary LOPs and Their Adverse Health Effects}

Despite the above serious considerations, many food science and nutritional researchers continue to reassure us of the "safety" of polyunsaturated fatty acid (PUFA)rich, vegetable-derived cooking oils when commonly employed for standard frying practices, and in general have reported that molecular indices commonly utilised for their safety evaluation are within permissible limits, e.g. [31]. Notwithstanding, the peroxidative deterioration of UFAs during such practices remains still largely unreported or even considered. But fortunately there is now an expanding group of scientists, along with members of the general public, who recognise the significance of circumventing or curtailing the use of PUFA-rich cooking oils for frying episodes, either domestically, or for commercial retail purposes in fastfood and other restaurants. Very high concentrations of LOPs are generated from exposure of these oils to high-temperature frying episodes, most especially cytotoxic, mutagenic and genotoxic aldehydes from these thermally-promoted, $\mathrm{O}_{2}$-driven, sequential autocatalytic UFA-damaging peroxidation activities. Unfortunately, such processes recycle when frying activities are repeated, as in frying oil reuse [19]. Further toxins generated include ketones, and epoxy-fatty acids such as leukotoxin $[5,32]$. In view of their transference into fried foods such as potato chips/French fries, these toxins are conveyed in a form which is available for human consumption.

To date, research results and data collected from a now almost overwhelming number of scientific papers and reports available have provided very powerful, almost profuse, evidence that substantially challenges those with viewpoints concordant with the negligible or limited health risk status of such PUFA-rich oils when used for frying purposes, and that any foods fried therein are "safe" for human consumption. However, currently only a small number of these developments have satisfactorily considered public health threats constituted by the ingestion of high concentrations of extremely toxic LOPs present in fried foods and other dietary sources. Evidence now available indicates that different classes of dietary LOPs, for example, saturated and $\alpha, \beta$-unsaturated aldehydes, and epoxy-fatty acids, are indeed absorbed from the gastrointestinal (GI) system into the systemic circulation $[5,33]$, a process facilitating their access to essential organs and tissues where they may induce cumulative, fried food diet-dependent chronic damage. Further reports available provide evidence that the ingestion of dietary LOPs may significantly contribute to enhanced risks of the development and/or perpetuation of CNCDs in humans, notably selected cancers, type 2 diabetes, and cardiovascular, inflammatory and neurodegenerative diseases, as indeed may excessive rates and extents of fried food consumption, as reviewed in [5]. Therefore, the major motif of the commentary presented in Ref. [5] is focused on the very wide range of potential public health threats presented by the human ingestion of primary LOPs (e.g. lipid hydroperoxides and epoxy-fatty acids), and their secondary fragmentation products (e.g., $\alpha, \beta$-unsaturated aldehydes, etc.), along with relevant considerations for COPs.

Intriguingly, diet serves as one of the most fundamental and modifiable risk elements for CNCDs, and the PURE study reported in [34] concluded that neither grand total, nor total SFA, MUFA and PUFA dietary intakes, were correlated with cardiovascular disease and myocardial infarction risks, nor cardiovascular disease mortality. Furthermore, evidence for negative relationships between SFA energy intake and non-cardiovascular disease and total mortalities, and stroke risk were established by these researchers. An inverse association between MUFA intake and total mortality was also found, and this is concordant with our observations that MUFA-rich cooking oils produce much lower concentrations of fried food-passive aldehydic LOPs than PUFAs during standard frying practices [5], and which is also consistent with two additional cohort studies which reported a diminishing association of total mortality with rising dietary MUFA energy intake (34, loc cit).

However, as is usually the case in such studies, the proportions of such FA classes ingested in forms which have been exposed to high-temperature frying or cooking episodes, and therefore LOP-contaminated, was completely overlooked in this investigation, as was the now established beneficial health effects offered by medium-chain length SFAs [35], rich sources of which are available in coconut oil.

Another recent study [32] found that exposure of a novel genetically-engineered algae-derived frying oil containing as much as $91 \%(\mathrm{w} / \mathrm{w})$ MUFAS and only $4 \%$ $(w / w)$ PUFAs generated markedly lower concentrations of aldehydic LOPs than those observed in PUFA-rich sunflower and corn oils during laboratory-simulated shallow frying episodes conducted at a temperature of $180^{\circ} \mathrm{C}$. As expected, when exposed to repetitive deepfrying episodes in a domestic fryer facility at $170^{\circ} \mathrm{C}(8$ $\mathrm{x}$ consecutive $10 \mathrm{~min}$. sessions), French fries fried in this MUFA-rich oil contained no ${ }^{1} \mathrm{H}$ NMR-detectable aldehydes, whereas those fried in sunflower oil under the same conditions had maximal $n$-alkanal, trans-2-alkenal and trans,trans-alka-2,4-dienal contents of ca. 2, 1 and 4 mmol./mol. French fry FA content respectively. Although hydroperoxide precursors of these aldehydes were detectable and quantified in the deep-fried sunflower oil product, these were absent from the MUFA-predominant algae oil, as indeed they were in corresponding French fry 
samples collected during this study. The latter observation presumably arises from their neutralization by natural potato biomolecules (and/or agents which result from their exposure to high-temperature frying episodes).

It may be informative to compare Materials Safety Data Sheet (MSDS) information [36] available for cholesterol with that of acrolein, a common secondary LOP found in fried foods. However, it is clear that cholesterol is difficult to evaluate from a toxicological standpoint; the MSDS states "data not available" for all sub-categories. Hazard symbols or risk phrases for it were also unavailable. Furthermore, in their toxicological categories, most, if not all listings from chemical companies and learned authorities provide the same statement. Indeed, reports on cholesterol toxicity remain extremely limited, and as we might expect, are generally restricted to accumulation of its unesterified form by macrophages, a process triggering advanced atherosclerosis [37, 38]. However, we should recall that low-density-lipoprotein (LDL), the apoprotein component of which can have key lysine residue sidechain amino functions "oxidatively" modified by reaction with free reactive aldehydes (known as oxLDL), these secondary LOPs presumably including those present in the circulation and which are dietary-derived [5]. In turn, oxLDL is uptaken by lesional macrophages via scavenger receptors, e.g., classes $\mathrm{A}$ and $\mathrm{CD} 36$, and cholesterol esters loaded in this manner are then transported to endo- or lysosomes where they are catalytically hydrolysed to their free cholesterol form [39].

In contrast, the aldehydic LOP acrolein carries the following MSDS warning: "Highly toxic if swallowed and by inhalation" both of which occur in a fast-food restaurant. "May cause corneal injury, severe eye irritation or burns to the eye. IARC Group 3 carcinogen. Lachrymator" [36]. $\mathrm{LD}_{50}$ (Lethal dose, 50\% fatality level) values reflect acute toxicity, a lethal dose causing $50 \%$ deaths within a 24 -hour period following administration. $\mathrm{LD}_{50}$ values reported for acrolein are 10.3 and $11.8 \mathrm{mg} / \mathrm{kg}$ for male and female rats respectively, and 13.9 and $17.7 \mathrm{mg} / \mathrm{kg}$ for male and female mice, respectively [40]. Interestingly, mice appear to be more resistant to this unsaturated aldehyde than rats, and evidence to support this was also found for crotonaldehyde [41]. Further information on the toxicological properties and recommended acceptable daily intake (ADI) values of selected aldehydic LOPs can be found in Refs. [5], [32] and [42].

In spite of all this information, are we to going to continue agonizing about cholesterol and ignore LOPs completely?

\section{The North Karelia Study in Finland (1972- 1997)}

Ischemic heart disease is undoubtably a multifactorial malady. Vartiainen [43] reported on the results of a long-term study of the Finnish population in North
Karelia, Finland, from 1972-1997. In this study, very impressive decreases were observed for three heartrelated indices: serum cholesterol, systolic and diastolic blood pressure and, most strikingly and, in our opinion, most significantly, tobacco smoking. Smoking imparts a wide range of hazardous free radical species into the human body. Additionally, cigarette smoke is known to contain high levels of aldehydes, in particular acrolein and its next highest homologue, crotonaldehyde [44]. Oxidative modification of lipid- and cholesterol-carrying low-density-lipoprotein (LDL) by secondary aldehydic LOPs so that they are recognized by macrophage scavenger receptors noted above, is a process which converts these cells to foam cells, the main constituents of atherosclerotic plaque and fatty streaks, as originally discovered by Steinberg's group in 1989 [45]. However, Singh [46] clearly demonstrated that smoking alters the blood lipid profile to a dyslipidemic state.

Prior to the commencement of the North Karelia-based research programme [43], in this region of Finland high intakes of butter, cheese, full-fat milk and cream were viewed by inhabitants to be particularly healthy foods, although they were, of course, believed to be responsible for the high mortality rates arising from cardiovascular diseases by researchers.

Advice provided by the investigators to the North Karelia population featured in Ref. [43] included the consumption of low- or non-fat, or sour milk rather than high-fat or whole milk products; reductions in the intakes of butter or margarine on bread, or use of soft margarine or soft butter (butter/oil mixtures) instead; the removal of visible fat in meat products when preparing them for cooking processes, the choice of only lean meat and sausages, and an advised preference for fish and poultry products rather than red meat; the preparation of food without adding animal fat, with a preference for boiling and baking (presumably rather than frying?); the employment of vegetable oil in salad dressings, and also during baking episodes; a major restriction on the consumption of egg yolks to only 2 per week; an enhanced whole-grain cereal intake; and finally, an increased consumption level of vegetables, roots, berries and fruits [43].

This whole population advice was indeed found to be highly valuable, and it certainly appears to have given rise to some extremely favourable outcomes. Interestingly, some aspects of these recommendations are also fully consistent with our postulates regarding the toxicological and adverse health effects of LOPs and COPs. For example, the major reductions in overall dietary fat consumption advised will undoubtedly include overall decreases in that of fried or other thermally-stressed oil-cooked foods, and hence LOP intake, if UFAs, and especially PUFAs, are contained in the frying oils utilized. In 2014, a survey of used cooking oils recovered for biofuel use in Finland had both palm and rapeseed oils as its major ingredients 
[47], and whereas both of these may generate LOPs when exposed to standard frying practices, their relatively high MUFA contents will be expected to retard this process somewhat. However, these vegetable oil sources appear to be at least partially ascribable to the advent and successful establishment and commercialization of global fast-food chains in that country during the span of the project from 1972 to 1997 . Moreover, the advice provided on increased intakes of antioxidant-rich vegetables, roots and berries, etc., may also serve as a potential LOP-suppressing bonus, both during food preparation and storage periods, and in vivo following ingestion. However, the above overall recommendation of using peroxidatively-degradable, LOP-generating UFA-containing vegetable oils in general for any high-temperature cooking processes may mitigate against this.

Notwithstanding, in contrast it should also be noted that although vegetable oil was used for cooking purposes by only $1-2 \%$ of the North Karelia population in 1972, this figure had escalated to ca. 34\% (reported as 'mainly vegetable oil') in 1997, with even higher reported increases in urban regions $[2,48]$. Hence, the contribution of SFAs towards energy intake was found to decline from 21 to $14 \%$ during the study period, whereas that from PUFAs increased from 3.5 to $5.0 \%$, although the latter observation appears to represent only a very small rise indeed [49]. Nevertheless, the [PUFA]:[SFA] concentration intake ratio markedly increased from 1972 to 1997, and the energy contribution from total lipids fell from 39 to $33 \%$ [50]. In view of the only very small increase in PUFA intake measured during the course of this lengthy programme (only $1.5 \%$ ), it appears that the vegetable oils used to replace animal fats or lard were largely the above MUFA-rich palm and rapeseed oil products [47] - as noted above, these are much more resistant to peroxidation than cooking oils which are loaded with PUFAs, such as sunflower and corn oils. Furthermore, as noted above, animal fats are rich in "heart-friendly" MUFAs as well as SFAs [6].

Finally, it should be noted that although the chemical instability of unsaturated nutrient lipids unfortunately leads to the concurrent ingestion of a range of cell- and DNA-damaging LOPs which arise from their oxidative degradation, the authors also do not doubt that an excessive dietary consumption level of SFAs may also serve to exert deleterious health effects, although their almost complete resistance to oxidation renders them valuable for use in high-temperature frying episodes, provided that the smoke-points of frying media employed are not exceeded. Indeed, one major focus of the current paper, and of some of the investigations considered herein, is how we might best avoid the adverse toxic effects posed by the dietary ingestion of LOPs. Moreover, there certainly also appears to be some public confusion and misconceptions regarding the term "fat calorie intake" expressed as a percentage of the total. Again, we do not dispute that the intake of high levels of "fat" will potentially lead to increased risks of a broad spectrum of adverse health effects, and that the human consumption of a more balanced diet will serve to circumvent or combat these hazards.

\section{Conclusion}

We have summarized the authors' collaborative views on the implications of dietary PUFAs and cholesterol, especially if subjected to conditions which promote their oxidation in foods to a series of toxic, health-threatening products, particularly high-temperature frying practices. Similar considerations apply when these food lipid substrates are stored at even ambient temperature, or when exposed to oxidation-promoting light sources for prolonged periods. The history associated with some common nonsensical misconceptions, for example the "health-promoting" properties of using PUFA-rich vegetable oils for frying processes, and the common and frequent bad press so unfairly received by the cholesterol molecule, has been critically discussed and reviewed here in some detail. Cholesterol is probably the most highly malefactor-targeted "bad-for-health" molecule ever, this despite being "innocent" of most, if not all such charges. Indeed, it certainly appears that some classical environmental poisons such as hydrogen cyanide and the herbicide paraquat receive much less attention.

For future public health-related studies, including epidemiological trials, a focus on the precise molecular nature of FA species rather than a consideration of only broad classifications of such macronutrients, for example as total SFAs, MUFAs, PUFAs, omega-3FAs, etc., is of much importance, and will undoubtedly serve to clarify such issues regarding the human intake of dietary LOPs and COPs, together with their potential adverse health effects. All too often, researchers involved in such investigations fail to recognize that only small modifications in defined molecular structure, including differential stereo- or geometric isomers of the same compound, may make an enormous difference to the biological and physiological effects of lipid macronutrients, and toxins such as aldehydic LOPs and COPs. Indeed, observation of the beneficial health effects exerted by odd-chain length FAs against incident type 2 diabetes by Forouhi et al. [30] serves as a good example of this, as do related data available on the generalised anti-inflammatory properties of medium-chain FAs (i.e., FAs of 8-12 carbon atom chain lengths, including capric, caprylic and lauric acids) as noted by Bengmark [35]. Moreover, our group has drawn attention to this issue in relation to coconut oil [51], which serves as a rich source of these medium-chain length FAs, as indeed does palm kernel oil. In the current study, the overall adverse consequences of the many erroneous historical conclusions regarding the 'safety' of dietary PUFAs, and "health hazardous" cholesterol have been reviewed in detail. However, the authors support the 
concept of a balanced diet, composed of all four traditional food groups. Moreover, we recognize the essentiality of omega- 3 and omega- 6 PUFAs. Nevertheless, now is the time to develop alternative, scientific evidence-based, viewpoints on these ubiquitous dietary agents, and which take into account the molecular stresses that they have been exposed to; these also include the adverse introduction of toxic contaminants via food processing techniques.

Data Availability: No datasets were employed to support this study. The Figures used to support study findings are included in the article.

Participant Consent: Not applicable.

Conflicts of Interest: Both authors confirm that they have no conflicts of interest.

Funding Statement: No funding was received for the undertaking of this project.

Acknowledgements: The authors are very grateful to Miles Gibson of De Montfort University, Leicester, UK for generating the $600 \mathrm{MHz} 1 \mathrm{H}$ NMR spectrum shown in Figure 3.

\section{References}

1. Anichkov. N. N. "Experimental Arteriosclerosis in Animals" in Arteriosclerosis: A Survey of the Problem. Ed. E. V. Cowdry, (New York, NY: McMillan), 1933, p. 271-322.

2. Anitschkow, N. and S. Chalatow. Uber experimentelle cholesterol-insteatose und ihre bedeutehung fur die entstehung einiger pathologischer prozesse. Zentralblat Allg Path Anat, 1913, 24(1): p. 1-9.

3. Smith, L. L. Cholesterol Autoxidation. (New York, NY: Plenum Press), 1981.

4. Guardiola, F. R., R. Codony, P. B. Addis, M. Rafecas, and J. Boatella. Biological effects of oxysterols: current status. Food Chem Toxicol. 1996, 34: p. 193211.

5. Grootveld, M., B. C. Percival, J. Leenders, and P. Wilson. Commentary - Potential adverse public health effects afforded by the ingestion of dietary lipid oxidation product toxins: Significance of fried food sources. Nutrients (Special Issue Targeted Nutrition in Chronic Disease) 2020, 12(4): 974; https://doi. org/10.3390/nu12040974
6. Brugiapaglia, A., C. Lussiana, G. Destefanis. Fatty acid profile and cholesterol content of beef at retail of Piemontese, Limousin and Friesian breeds. Meat Sci. 2014, 96(1): p. 568-573, ISSN 0309-1740, https://doi.org/10.1016/j.meatsci.2013.08.012.

7. Dinamarca, E., F. Garrido, A. Valenzuela. Simple high vacuum distillation equipment for deodorizing fish oil for human consumption. Lipids 1990, 25: p. 170-171. https://doi.org/10.1007/BF02544333

8. Mohanty, B., Hauzoukim, S. Swain, S. Swain. Fish oil's beneficial effect to human health: A review. Biosc Biotech Res Comm. Special Issue 2020, 13(12): p. 10-14.

9. Park, S. W. and P. B. Addis. Identification and quantitative estimation of oxidized cholesterol derivatives in heated tallow. J Agric Food Chem. 1986, 34: p. 653-659.

10. Park, S. W. and P. B. Addis. Further investigation of oxidized cholesterol derivatives in heated fats. J Food Sci. 1986, 51: p. 1380-1381.

11. Keys, A. in Kromhout, D., Menotti, A. and Blackburn, H, (eds.) (1993). The Seven Countries Study: A Scientific Adventure in Cardiovascular Disease Epidemiology (Utrecht, The Netherlands: Brouwer Offset bv). p. 16-25.

12. Keys, A. The cholesterol problem. Voeding 1952, 13: p. 539-555.

13. Keys, A. (ed), Seven Countries: A multivariate analysis of death and coronary heart disease, 1980. (Cambridge, Mass.: Harvard University Press). ISBN 0-674-80237-3

14. Papuc, C., G. V. Goran, C. N. Predescu and V. Nicorescu. (2017), Mechanisms of oxidative processes in meat and toxicity induced by postprandial degradation products: A review. Comp Rev Food Sci Food Safety 2017, 16: p. 96-123. https://doi. org/10.1111/1541-4337.12241

15. Teicholz, N. “The Big Fat Surprise” (New York, NY: Simon and Schuster), 2014.

16. de Souza, R. J., A. Mente, A. Maroleanu, A. I. Cozma, V. Ha, T. Kishibe, et al. Intake of saturated and trans unsaturated fatty acids and risk of all cause mortality, cardiovascular disease, and type 2 diabe- 
tes: systematic review and meta-analysis of observational studies $B M J$ 2015, 351: h3978 doi:10.1136/ bmj.h3978

17. Esterbauer, H. "Aldehydic products of lipid peroxidation" in Free Radicals, Lipid Peroxidation and Cancer Eds. D. C. H. McBrien and T. F. Slater, (London: Academic Press), p. 101-128.

18. Esterbauer, H. R. Schaur and H. Zollner. Chemistry and biochemistry of 4-hydroxynonrnal, malonaldehyde, and related aldehydes. Free Rad Biol Med. 1991, 11: p. 81-128.

19. Le Gresley, A., G. Ampem, S. De Mars, M. Grootveld, D. P. Naughton. Real-world" evaluation of lipid oxidation products and trace metals in French fries from two chain Fast-food restaurants. Frontiers Nutr. 2021, 8:(17 pages). URL=https://www. frontiersin.org/article/10.3389/fnut.2021.620952. DOI $=10.3389 /$ fnut.2021.620952. $\quad$ ISSN=2296$-861 \mathrm{X}$

20. Indart, A., M. Viana, M. C. Grootveld, C. J. L. SiIwood., I. Sanchez-Vera and B. Bonet. Teratogenic actions of thermally-stressed culinary oils in rats. Free Rad Res. 2002, 36(10): p. 1051-1058.

21. Grootveld, M., C. J. L. Silwood, P. B. Addis, A. W. D. Claxson, B. Bonet Serra and M. Viana. Health effects of oxidized heated oils. Foodservice Res Intern. 2001, 13: p. 41-55.

22. Addis, P. B., T. P. Carr, C. A. Hassel, Z. Z. Huang and G. J. Warner. Atherogenic and anti-atherogenic factors in the human diet. Biochem. Soc. Symp.1995, 61: p. 259-271.

23. Pryor, W. A. Vitamin E and heart disease: basic science to clinical intervention trials. Free Rad Biol Med. 2000, 28: p. 141-164.

24. Lawrence, G. D. Dietary fats and health: dietary recommendations in the context of scientific evidence. Adv Nutr. 2013, 4(3): p. 294-302. doi: 10.3945/an.113.003657. PMID: 23674795; PMCID: PMC3650498.

25. Lecerf, J. and M. De Lorgeril. Dietary cholesterol: From physiology to cardiovascular risk. Brit J Nutr. 2011, 106(1): p. 6-14. doi:10.1017/ S0007114511000237
26. Apfelbaum, M. Which is the nutritional advantage of the consumption of a free-cholesterol butter? Rev Prat. 1992, 42: p. 1925-1926.

27. McNamara, D. J. The fifty year rehabilitation of the egg. Nutrients. 2015, 7(10): p. 8716-8722. doi:10.3390/nu7105429.

28. DiMarco, D. M., G. H. Norris, C. L. Millar, C. N. Blesso, M. L. Fernandez. Intake of up to 3 eggs per day is associated with changes in HDL function and increased plasma antioxidants in healthy, young adults. J Nutr. 2017, 147(3): p. 323-29.

29. Salas-Salvado, J., et al. Reduction in the incidence of type 2 diabetes with the Mediterranean diet: results of the PREDIMED-Reus nutrition intervention randomized trial. Diabetes Care. 2011, 34(1): p. $14-19$.

30. Forouhi, N. G., A. Koulman, S. J. Sharp et al. Differences in the prospective association between individual plasma phospholipid saturated fatty acids and incident type 2 diabetes: the EPIC-InterAct case-cohort study. Lancet Diabetes Endocrinol. 2014. http://dx.doi.org/10.1016/S2213-8587(14)70146-9

31. Opperman, M., A. Benadé, C. Abrecht, L. Matsheka. South African seed oils are safe for consumption. S Afr J Clin Nutr. 2016, 29(1): p. 7-11.

32. Moumtaz, S., B. C. Percival, D. Parmar, K. L. Grootveld, P. Jansson and M. Grootveld. Generation of toxic $\alpha, \beta$-unsaturated and saturated aldehydes during simulated shallow frying episodes: comparisons of common frying oils with a novel high-stability algae oil product. Sci Rep. 2019, 9 (Article number 4125): 21 pages.

33. Grootveld, M., M. D. Atherton, A. N. Sheerin, J. Hawkes, D. R. Blake, T. E. Richens et al. In vivo absorption, metabolism, and urinary excretion of $\alpha, \beta$-unsaturated aldehydes in experimental animals. Relevance to the development of cardiovascular diseases by the dietary ingestion of thermally stressed polyunsaturate-rich culinary oils. J. Clin. Invest. 1998, 101(6): p. 1210-1218.

34. Dehghan, M., A. Mente, X. Zhang, S. Swaminathan, W. Li, Mohan, V. et al. Associations of fats and carbohydrate intake with cardiovascular disease and mortality in 18 countries from five conti- 
nents (PURE): a prospective cohort study. Lancet 2017, 390: p. 2050-2062.

35. Bengmark, S. Choose right carbohydrates and right fats (RCRF) - keys to optimal health. Hepatobiliary Surg Nutr. 2017, 6: p. 429-433.

36. ht t p s:// r.se a r ch.yahoo.com / ylt=AwrIQhI3iyphtOAAhip3Bwx.;_ylu=Y29 sbwMEcG9zAzEEdnRpZANDMjAxN 18xBH NlYwNzcg- $-/ R V=2 / R E=1630206903 / R O=10 /$ $\mathrm{RU}=\mathrm{https} \% 3 \mathrm{a} \% 2 \mathrm{f} \% 2 \mathrm{fwww} . \mathrm{msd}$.com $\% 2 \mathrm{f} / \mathrm{RK}=2 /$ $\mathrm{RS}=0 \mathrm{GmwEQ} 9 \mathrm{MLeVFAcrJ5VarHw51soQ-}$

37. Kellner-Weibel, G., Y. J. Geng, G. H. Rothblat. Cytotoxic cholesterol is generated by the hydrolysis of cytoplasmic cholesteryl ester and transported to the plasma membrane. Atherosclerosis 1996, 146: p. 309-319.

38. Zhang, K., R. Kaufman. Unfolding the toxicity of cholesterol. Nat Cell Biol. 2003, 5: p. 769-770. https://doi.org/10.1038/ncb0903-769

39. Xu, X., A. Zhang, N. Li, P. L. Li and F. Zhang. Concentration-dependent diversifcation effects of free cholesterol loading on macrophage viability and polarization. Cell Physiol Biochem. 2015, 37(2): p. 419-431. doi:10.1159/000430365

40. Directive 98/8/EC concerning the placing of biocidal products on the market. Inclusion of active substances in Annex I to Directive 98/8/EC Assessment Report. Competent Authority Report: UK ACROLEIN PT12 October 2009.

41. Wolfe, G., M. Rodwin, J. French, G. Parker. Thirteen week subchronic toxicity study of crotonaldehyde (CA) in F344 rats and B6C3F1 mice. Toxicologist 1987, 7: p. 209.

42. Grootveld, M., B. C. Percival, S. Moumtaz, M. Gibson, K. Woodason, A. Akhtar et al. Commentary: Iconoclastic reflections on the 'safety' of polyunsaturated fatty acid-rich culinary frying oils: Some cautions regarding the laboratory analysis and dietary Ingestion of lipid oxidation product toxins. Appl Sci. 2021, 11(5): 2351 (34 pages). https://doi. org/10.3390/app11052351

43. Vartiainen, E. The North Karelia Project: Cardiovascular disease prevention in Finland. Glob Car- diol Sci Pract. 2018, (2): p. 13.

44. RIVM report 340630002/2006. Adverse health effects of cigarette smoke: aldehydes crotonaldehyde, butyraldehyde, hexanal, and malonaldehyde. van Andel I, Sleijffers A, Schenk E, Rambali E, Wolterink G, van de Werken G, van Aerts LAGJM, Vleeming W, van Amsterdam JGC. (2006)

45. Steinberg, D., S. Parthasarathy, T. E. Carew, J. E. Khoo and Witztum, J. L. Beyond cholesterol. Modifications of low-density lipoprotein that increase its atherogenicity. N Engl J Med. 1989, 320(14): p. 915-924. doi: 10.1056/NEJM198904063201407. PMID: 2648148.

46. Singh, D. Effect of cigarette smoking on serum lipid profile in male population of Udaipur. Biochem Anal Biochem. 2016, 5:3. https://doi. org/10.4172/2161-1009.1000283

47. https://r.search.yahoo.com/_ylt=AwrIS. W27 Kxg4jIAswZ3Bwx.;_ylu=Y29sbwME cG9zAzEEdnRpZAMEc2 VjA $3 \mathrm{Ny} / \mathrm{RV}=2$ / $\mathrm{RE}=1621974326 / \mathrm{RO}=10 / \mathrm{RU}=\mathrm{https} \% 3 \mathrm{a} \% 2 \mathrm{f} \% 2$ fadvancedbiofuelsusa.info $\% 2$ fuco-collection-and-use-in-finland $\% 2 \mathrm{f} / \mathrm{RK}=2 / \mathrm{RS}=$ _NYZ72sHeayoKnGcmBRg6O7U8mQ-

48. Vartiainen, E., T. Laatikainen, H. Tapanainen and P. Puska. Changes in serum cholesterol and diet in North Karelia and all Finland. Global Heart. 2016, 11: p. 179-184.

49. Helakorpi, S., A. Uutela, R. Prattala and P. Puska. Health behaviour among Finnish adult population. 1998. Publication B10/1998. Helsinki: National Public Health Institute.

50. Pekka, P., P. Pirjo and U. Ulla. Part III. Can we turn back the clock or modify the adverse dynamics? Programme and policy issues. Influencing public nutrition for non-communicable disease prevention: from community intervention to national programme - experiences from Finland. Pub Health Nutr. 2002, 5(1A): p. 245-251. DOI: 10.1079/ PHN2001300.

51. Grootveld, M., B. C. Percival and K. Grootveld. Chronic non-communicable disease risks presented by lipid oxidation products in fried foods. $\mathrm{He}$ patobiliary Surg. Nutr. 2018, 7(4): p. 305-312. doi: 10.21037/hbsn.2018.04.01. 